\title{
Stochastic Optimal Investment under Inflationary Market with Minimum Guarantee for DC Pension Plans
}

\author{
Othusitse Basimanebotlhe $\mathrm{e}^{1,2} \&$ Xiaoping Xue ${ }^{1}$ \\ ${ }^{1}$ School of Science, Department of Mathematics, Harbin Institute of Technology, Harbin, China \\ ${ }^{2}$ Faculty of Science, Department of Mathematics, University of Botswana, Gaborone, Botswana \\ Correspondence: Othusitse Basimanebotlhe, School of Science, Department of Mathematics, Harbin Institute of \\ Technology, Harbin, 150001, Nangang District, China E-mail: ot_bas@yahoo.co.uk \\ Received: April 8, 2015 Accepted: May 3, 2015 Online Published: July 11, 2015 \\ doi:10.5539/jmr.v7n3p1 URL: http://dx.doi.org/10.5539/jmr.v7n3p1
}

\begin{abstract}
The paper studies the optimal investment strategies to partake in a defined contribution (DC) pension fund, with the expected minimum guarantee process. The pension fund manager aspires to maximize the surplus, where his benefit lies in a complete market that is subjected to inflation rate. There are only three assets of investment being; the non risky asset and two risky assets. The dynamics of the wealth in our model takes into account a certain proportion of the client's salary paid as the contribution towards the pension fund and any other extra amount paid to amortize the fund.

Applying the method of stochastic optimal control to the portfolio management problem, a non-linear second order differential equation for the value function was derived. A constant risk relative aversion (CRRA) utility function was considered to obtain the explicit solutions for the optimal investment strategies. Finally, a numerical simulation is presented to illustrate the behaviour of the model.
\end{abstract}

Keywords: stochastic optimal control, defined contribution pension fund, Hamilton-Jacobi-Bellman equation; Geometric Brownian motion

\section{Introduction}

The recent economic developments as mentioned in (Beletski, 2006), have shown that neither equity nor interest rates have been effective in protecting customers against purchasing power erosion. This brings the concept of inflation to play. The concept of inflation has been studied in actuarial sciences and in macro economics which can be traced as far back as the work of (Fisher, 1930) who studied, The Theory of Interest. It is the work of (Fisher, 1930) which formed the famous hypothesis that the difference between the nominal and real interest rates should vary closely with the movements in expected inflation. The inflation rate would be considered as a geometric Brownian motion in this paper.

A similar problem of a Defined Contribution (DC) pension fund in the presence of minimum guarantee has been discussed by (Deelstra et al, 2003). In (Deelstra et al, 2003) the fund manager invest the initial wealth and the stochastic contribution flow into the financial market, where the stochastic interests followed the Cox-IngersollRoss (CIR) model. Of recent (Nkeki \& Nwozo, 2012) and (Nkeki \& Nwozo, 2013) have studied the optimal portfolio strategies with minimum guarantee and protection in a DC scheme. In (Nkeki, 2012), the author's aim was to obtain the optimal share of portfolio values that depends on the minimum guarantee. It was found that a certain proportion of wealth has to be transfered into the cash account from the indexed bonds and stock portfolios in-order to cushion the inflation associated with the pension plan member's portfolios.

The pension fund managers' problem has been choosing the best investment strategies, which has gained more interest from other researchers like (Boulier et al, 2001), (Vigna \& Haberman, 2001), (Haberman \& Vigna, 2002), (Cairns et al, 2006), just to mention a few. When a pension fund manager calculates the best strategies to optimize future rewards he has to consider the actuarial liabilities and options embedded in a pension as outlined in (Wilkie, 1985), (Blake, 1998) in (Boulier et al, 2001).

The aim of this paper is to find the optimal investment strategies that the fund manager has to deploy to maximize his/her rewards or benefits. It must be noted that the benefits of the fund manager lies in the surplus amount as in (Deelstra et al, 2003). Unlike other researchers such as (Devolder et al, 2003),(Deelstra et al, 2003), (Deelstra et $a l, 2000)$ where the risk was only due to stock price market, in this work we include the risk associated with 
the inflation. Our work is similar to the study of (Gao, 2009), the difference between our work and that of (Gao, 2009), is on the number of assets that the fund manager trade upon. We considered the risky assets to be following the Geometric Brownian motion (GBM), whilst in (Gao, 2009), they were modeled to be of constant elasticity of variance $(\mathrm{CEV})$. The other aspect that distinguishes this study from the works of other researchers is the inclusion of a certain amount of contribution, known as the supplementary contribution other than the agreed amount with the employer. The supplementary contribution is incurred by the client in order to amortize past and present imbalances in the fund that may be due to inflation, change of policies or any other factors. The CRRA utility function is considered as the function whereby the manager aspires to maximize his rewards. The minimum guarantee would simultaneously acts as the solvency level to our problem.

\section{Financial Model Formulation}

We consider a continuous trading economy over the time period $[0, T]$ characterized by the 2-dimensional Brownian motions $\left(W^{S}(t), W^{I}(t)\right)$, defined on a given filtered probability space $\left(\Omega, \mathcal{F}, \mathcal{F}_{t}^{S}, \mathcal{F}_{t}^{I}, \mathbb{P}\right)$ where $\mathbb{P}$ is the real world probability measure. $\left\{\mathcal{F}_{t}^{I}, \mathcal{F}_{t}^{S}\right\}$ are right continuous filtrations whose informations are generated by the two standard Brownian motions $\left(W^{S}(t), W^{I}(t)\right)$ whose sources of uncertainties are respectively to the inflation rate and the stock market. The market is assumed to be well defined within interval time, $t \in[0, T]$, where $T$ is the terminal time period. It is assumed that the investor has three assets to trade upon, being: the cash account (risk-less), the stock price and the inflation-linked bond. The correlation between the two Brownian motions is given by $d W_{I} d W_{S}=\frac{1}{2} \rho_{S I} d t$.

(i) The state variables are chosen as assets or the wealth of the pension plan at any time $t$, that is;

$$
X(t), \quad(t \in[0, T])
$$

(ii) The decision variables are;

$$
\left\{u_{S}(t), u_{B}(t)\right\}, \quad(t \in[0, T])
$$

are the proportions to be invested in the stock price and inflation-linked bond.

Proposition 1. The inflation rate is given by the equation:

$$
\frac{d I(t)}{I(t)}=\pi_{e}(t) d t+\sigma_{I} d W_{I}, \quad I(0)=i
$$

whose solution is given by

$$
I(t)=i \exp \left\{\int_{0}^{t} \pi_{e}(s) d s-\frac{1}{2} \sigma_{I}^{2} t+\sigma_{I} W_{I}(t)\right\}
$$

for $\pi_{e}(t)=r_{N}(t)-r_{R}(t)$ as the expected inflation rate which is the difference between the nominal, $\left(r_{N}\right)$ and real $\left(r_{R}\right)$ interest rate. The constant volatility of the inflation is represented by $\sigma_{I}$.

As for the proof refer to (Zhang et al, 2007).

The real interest rate through out this paper is described by the stochastic differential equation,

$$
d r_{R}(t)=\left(\alpha-b r_{R}(t)\right) d t+\sigma_{R} d W_{R}(t)
$$

where the parameter $\sigma_{R}$ represents the instantaneous volatility of the real interest rate. The parameter $\alpha$ denotes the long term mean level, while $\beta$ represents the rate of mean reversion.

The pension fund manager has three assets to trade upon, the two risky assets being the stock, the inflation-linked bond and lastly the risk-less asset (bank account).

The risk-less asset is denoted by $\left(S_{0}(t)\right)$, and evolves according to the equation:

$$
\frac{d S_{0}(t)}{S_{0}(t)}=r_{R}(t) d t
$$

with initial condition $S_{0}(0)=1$.

Proposition 2. The stock price subject to inflation evolves according to the Itô process:

$$
\frac{d S(t)}{S(t)}=\left(r_{R}(t)+\lambda_{1} \sigma_{S}^{S}+\lambda_{2} \sigma_{S}^{I} \theta_{I}\right) d t+\sigma_{S}^{S} d W_{S}+\sigma_{S}^{I} d W_{I}
$$


that has the solution:

$$
\begin{aligned}
S(t)=\exp \left\{\int_{0}^{t} r_{R}(s) d s+\left(\lambda_{1} \sigma_{S}^{S}+\lambda_{2} \sigma_{S}^{I} \theta_{I}-\frac{1}{2}\left[\left(\sigma_{S}^{S}\right)^{2}\right.\right.\right. & \left.\left.+\left(\sigma_{S}^{I}\right)^{2}+\rho_{S I} \sigma_{S}^{I} \sigma_{S}^{S}\right]\right) t \\
& \left.+\sigma_{S}^{I} W_{I}(t)+\sigma_{S}^{S} W_{S}(t)\right\}
\end{aligned}
$$

with $S(0)=1$ and $\theta_{I}$ denoting the inflation price market risk. The constants $\lambda_{1}$ and $\lambda_{2}$ are the instantaneous risk premiums associated respectively with the positive volatility constants, $\sigma_{S}^{S}$ and $\sigma_{S}^{I}$ as mentioned in (Deelstra et al, 2000).

Proof. Let

$$
f(t, S(t))=\log S(t)
$$

Using Itô's lemma, we have that

$$
d(\log S(t))=\left[\left(r_{R}(t)+\lambda_{1} \sigma_{S}^{S}+\lambda_{2} \sigma_{S}^{I} \theta_{I}\right) d t+\sigma_{S}^{S} d W_{S}+\sigma_{S}^{I} d W_{I}\right]+\frac{1}{2}\left[\frac{-1}{S^{2}(t)}\right][d S(t)]^{2}
$$

It can be similarly shown that

$$
[d S(t)]^{2}=\left[\left(\sigma_{S}^{S}\right)^{2}+\left(\sigma_{S}^{I}\right)^{2}+2 \sigma_{S}^{I} \sigma_{S}^{S} d W_{I} d W_{S}\right] S^{2}(t)
$$

We know that the correlation between the two Brownian motions is given as

$$
d W_{I} d W_{S}=\frac{1}{2} \rho_{S I} d t
$$

Therefore, substituting equation (9) into equation (8) and replacing the results into equation (7), we get:

$$
\begin{array}{r}
d(\log S(t))=\left[\left(r_{R}(t)+\lambda_{1} \sigma_{S}^{S}+\lambda_{2} \sigma_{S}^{I} \theta_{I}\right) d t+\sigma_{S}^{S} d W_{S}+\sigma_{S}^{I} d W_{I}\right] \\
-\frac{1}{2}\left[\left(\sigma_{S}^{S}\right)^{2}+\left(\sigma_{S}^{I}\right)^{2}+\sigma_{S}^{I} \sigma_{S}^{S} \rho_{S I}\right] d t .
\end{array}
$$

Integrating both side of the equation over the interval $[0, t]$,

$$
\begin{aligned}
\log S(t)=\left[\int_{0}^{t} r_{R}(s) d s+\left(\lambda_{1} \sigma_{S}^{S}+\right.\right. & \left.\left.\lambda_{2} \sigma_{S}^{I} \theta_{I}\right) t+\sigma_{S}^{S} W_{S}(t)+\sigma_{S}^{I} W_{I}(t)\right] \\
& -\frac{1}{2}\left[\left(\sigma_{S}^{S}\right)^{2}+\left(\sigma_{S}^{I}\right)^{2}+\sigma_{S}^{I} \sigma_{S}^{S} \rho_{S I}\right] t
\end{aligned}
$$

Thus,

$$
\begin{aligned}
S(t)=\exp \left[\int_{0}^{t} r_{R}(s) d s+\left[\left(\lambda_{1} \sigma_{S}^{S}+\lambda_{2} \sigma_{S}^{I}\right)\right.\right. & \left.-\frac{1}{2}\left[\left(\sigma_{S}^{S}\right)^{2}+\left(\sigma_{S}^{I}\right)^{2}+\sigma_{S}^{I} \sigma_{S}^{S} \rho_{S I}\right]\right] t \\
& \left.+\sigma_{S}^{S} W_{S}(t)+\sigma_{S}^{I} W_{I}(t)\right]
\end{aligned}
$$

The last asset is the inflation-linked bond that has the following relation with the inflation index, using the idea of (Zhang et al, 2007) and (Bodie et al, 2011).

$$
\frac{d B(t, I(t))}{B(t, I(t))}=\left(r_{N}(t) d t+\frac{d I(t)}{I(t)}\right.
$$

Proposition 3. The inflation linked bond is described the stochastic differential equation (SDE):

$$
\frac{d B(t, I(t))}{B(t, I(t))}=\left(r_{R}(t)+\sigma_{I} \theta_{I}\right) d t+\sigma_{I} d W_{I}
$$

which has the solution

$$
B(t, I(t))=B(0, I(0)) \exp \left\{\int_{0}^{t} r_{R}(s) d s+\left(\sigma_{I} \theta_{I}-\frac{1}{2}\left(\sigma_{I}\right)^{2}\right) t+\sigma^{I} W_{I}(t)\right\}
$$


Proof. Let

$$
f(t, B(t, I(t)))=\log B(t, I(t))
$$

Using Itô's lemma, we get;

$$
\begin{array}{r}
d(\log B(t, I(t)))=\left[\left(r_{R}(t)+\sigma_{I} \theta_{I}\right) d t-\sigma_{I} d W_{I}\right] \\
+\frac{1}{2}\left[\frac{-1}{B^{2}(t, I(t))}\right]\left(\sigma_{I}\right)^{2} B^{2}(t, I(t)) d t .
\end{array}
$$

Hence, by integration over the interval $[0, t]$ we have;

$$
\log B(t, I(t))=\int_{0}^{t}\left(r_{R}(t)+\sigma_{I} \theta_{I}\right) d t-\frac{1}{2}\left(\sigma_{I}\right)^{2} t+\sigma_{I} W_{I}(t)
$$

Taking the exponential at the initial condition $B(0, I(0))$, finally yields

$$
B(t, I(t))=B(0, I(0)) \exp \left\{\int_{0}^{t} r_{R}(s) d s+\left(\sigma_{I} \theta_{I}-\frac{1}{2}\left(\sigma_{I}\right)^{2}\right) t+\sigma_{I} W_{I}(t)\right\}
$$

which is our desired results.

The proportions to be invested in the stock, inflation-linked bond and the account are respectively $u_{S}(t), u_{B}(t)$ and $1-u_{S}(t)-u_{B}(t)$. The pension fund manager's main task is to try to identify the best strategies of investment among the three assets.

\subsection{Salary}

The wage of the pension fund contributor or client is described by the SDE:

$$
d P(t)=\mu_{p}(t) P(t) d t+\sigma_{p}^{S} P(t) d W_{S}(t)+\sigma_{p}^{I} P(t) d W_{I}(t)
$$

where $\mu_{p}(t)$ is the expected instantaneous rate of the salary. The two volatility scale factors of the stock and inflation are denoted by $\sigma_{p}^{S}$ and $\sigma_{p}^{I}$ respectively.

\subsection{Contribution Process}

The client has to contribute a certain proportion of his/her salary, and it evolves according to the equation;

$$
c(t)=\delta P(t)+\xi(t), \quad \text { for } t \in[0, T]
$$

where $\delta P(t)$ is the proportion of the salary that the client or employee has agreed upon with the employer to be paid towards the pension. The function $\xi(t)$ is a supplementary contribution paid to amortize past and present experiences deviations. The supplementary contribution is a deterministic function given by

$$
d \xi(t)=-I \xi(t) \quad \text { for } t \in[0, T]
$$

where $I$ the known inflation rate at that particular time. It is assumed that a large sum amount of money, $X(0)=X_{0}$ was initially deposited into the pension at time $t=0$. The contribution is a non negative, progressive process such that,

$$
\int_{0}^{T} c(t) d t<\infty, \quad \mathbb{P}-\text { a.s., } \forall t \in[0, T]
$$

\subsection{Minimum Guarantee}

The minimum guarantee at anytime, $(t \in[0, T])$ described by the equation;

$$
m(t)=\int_{0}^{t} f(\tau) c(\tau) e^{I(T-\tau)} d \tau
$$


Therefore, the expected minimum guarantee process as outlined earlier in (Nkeki \& Nwozo, 2013) it is given by the definition below.

Definition 1 The value of the expected minimum guarantee process is defined as,

$$
\begin{aligned}
H(t) & =E_{t}[m(t)] \\
& =E_{t}\left[\int_{0}^{t} f(\tau) c(\tau) \exp [I(T-\tau)] d \tau\right], \quad t \geq 0
\end{aligned}
$$

where $E_{t}$ is the conditional expectation with respect to the filtrations, $\left\{\mathcal{F}_{t}^{I}, \mathcal{F}_{t}^{S}\right\}_{t \geq 0}$. The function $f(t)$ is a discounting factor that adjust the real interest rate to the market price risks.

The discounting factor $f(t)$ is given by stochastic differential equation

$$
d f(t)=f(t)\left(r_{R}(t) d t-\theta^{T} d W(t)\right)
$$

where $d W(t)=\left(\begin{array}{l}d W_{S}(t) \\ d W_{I}(t)\end{array}\right), \theta^{T}=\left(\begin{array}{ll}\theta_{I} & \theta_{S}\end{array}\right)$ and $\theta_{S}$ denotes the stock market price risk. From equation (25) under definition (1), we have the following lemma.

Lemma 1. The expected minimum guarantee process is proportional to the the contribution process, that is;

$$
H(t)=\frac{c(t) \exp [I T]}{\Psi^{\prime}(t)}[\exp [\Psi(t)]-1], \quad \forall t \geq 0 .
$$

where

$$
\Psi^{\prime}(t)=\int_{0}^{t} r_{R}(t) d t-\frac{1}{2}\left(\left\|\theta_{I}\right\|-2 I\right)^{2} t
$$

proof. Using definition (1), the expected minimum guarantee process is,

$$
H(t)=E_{t}\left[\int_{0}^{t} f(s) c(s) \exp [I(T-s)] d s\right], \quad s \in(0, t) .
$$

The contribution process is independent from both filtrations $\left\{\mathcal{F}_{t}^{I}, \mathcal{F}_{t}^{S}\right\}_{t \geq 0}$, hence,

$$
H(t)=c(t) \exp [I T] E_{t}\left[\int_{0}^{t} f(s) \exp [-I s] d s\right], \quad s \in(0, t) .
$$

substituting the stochastic discount factor as given in equation (26), into the above equation (28) we have:

$$
\begin{array}{r}
H(t)=c(t) \exp [I T] \int_{0}^{t} E_{t}\left[\operatorname { e x p } \left[\int_{0}^{s} r_{R}(\tau) d \tau-\frac{1}{2}\left(\left\|\theta_{I}\right\|^{2}-2 I\right) s\right.\right. \\
\left.\left.-\theta_{I}^{T} W_{I}(s)\right]\right]
\end{array}
$$

By taking the conditional expectation, we get

$$
H(t)=c(t) \exp [I T]\left[\int_{0}^{t} \exp \left[\int_{0}^{s} r_{R}(\tau) d \tau-\frac{1}{2}\left(\left\|\theta_{I}\right\|^{2}-2 I\right) s\right]\right], \quad s \in(0, t)
$$

which completes the proof as it yields,

$$
H(t)=\frac{c(t) \exp [I T]}{\Psi^{\prime}(t)}[\exp [\Psi(t)]-1], \quad t \geq 0
$$

where

$$
\Psi^{\prime}(t)=\int_{0}^{t} r_{R}(\tau) d \tau-\frac{1}{2}\left(\left\|\theta_{I}\right\|^{2} t-2 I\right) t
$$




\subsection{Wealth}

Let $X(t)$ denote the wealth of the fund at any time, $(t \in[0, T])$. Taking into consideration equations (4), (5) and (14), the wealth process is described by the SDE:

$$
\begin{aligned}
& \left.d X(t)=\left(\left[\begin{array}{ll}
r_{R}(t)+\left(u_{S}(t)\right. & u_{B}(t)
\end{array}\right)\left(\begin{array}{c}
\lambda_{1} \sigma_{S}^{S}+\lambda_{2} \sigma_{S}^{I} \theta_{I} \\
\sigma_{I} \theta_{I}
\end{array}\right)\right] X(t)+c(t)\right) d t \\
& +\left(u_{S}(t) \quad u_{B}(t)\right)\left(\begin{array}{cc}
\sigma_{S}^{S} & \sigma_{S}^{I} \\
0 & \sigma_{I} \theta_{I}
\end{array}\right)\left(\begin{array}{l}
d W_{S}(t) \\
d W_{I}(t)
\end{array}\right) X(t)+u_{B}(t) \sigma^{I} X(t) d W_{I}
\end{aligned}
$$

The decision variables are $\left(u_{S}(t) \quad u_{B}(t) \quad 1-u_{S}(t)-u_{B}(t)\right) \equiv \mathcal{U}(\cdot) \in \mathcal{U}_{a d}(\cdot)$, where $\mathcal{U}_{a d}(\cdot)$ represents a set of admissible control variables. The rate of wealth whenever the volatility constants, $\sigma_{(\cdot)} \equiv 0$ becomes a deterministic equation given by;

$$
\begin{cases}\left.d X(t)=\left(\left[\begin{array}{ll}
r_{R}(t)+\left(u_{S}(t)\right. & u_{B}(t)
\end{array}\right)\left(\begin{array}{cc}
\lambda_{1} \sigma_{S}^{S}+\lambda_{2} \sigma_{S}^{I} \theta_{I} \\
\sigma_{I} \theta_{I}
\end{array}\right)\right] X(t)+c(t)\right) d t, & t<T \\
X(T)=x \geq H_{T}, & t=T\end{cases}
$$

where $\sigma_{(\cdot)}$ denotes $\left(\begin{array}{ccc}\sigma_{S}^{S} & \sigma_{S}^{I} & \sigma_{I}\end{array}\right)^{T}$ and $H_{T}$ is the expected terminal minimum guarantee which also acts as the solvency level. The solvency level constrains the manager from enacting arbitrary strategies which may lead to bankruptcy. To consider the stochastic case of the wealth, we assume that $\sigma_{(\cdot)}$ are positive constants.

Lemma 2. Given any $x \geq H(t)$, at anytime $t \in[0, T]$. The set of admissible controls $\mathcal{U}_{a d}(\cdot)$ is non empty if and only if $\mathcal{U}(\cdot) \equiv 0$ is admissible. This occurs whenever

$$
x \geq \frac{-c(t)}{r_{R}(t)}, \quad \forall t \leq T .
$$

Precisely, the set of admissible controls $\mathcal{U}_{a d}(\cdot)$ is non empty for every $x \geq H(t)$ if and only if

$$
H(t) \geq \frac{-c(t)}{r_{R}(t)}, \quad \forall t \leq T .
$$

proof Let $x \geq H(t), \forall t \geq 0$. It is clear that if $\mathcal{U}(\cdot) \equiv 0$ is admissible at $(T, x)$, then the set of admissible controls $\mathcal{U}_{a d}(\cdot)$ is non empty.

Let $\mathcal{U}(\cdot)$ be an admissible strategy and set the ordinary differential equation $X(t)=X(t, T, \mathcal{U}(\cdot), x)$. Under the new probability measure $\tilde{\mathbb{P}}$ which also depends on $t$ and is defined under the filtrations $\left\{\mathcal{F}_{t}^{I}, \mathcal{F}_{t}^{S}\right\}$. The new probability measure is defined by the exponential process that is assumed to be a martingale in $\mathbb{P}$, that is;

$$
\tilde{\mathbb{P}}=\exp \left(\theta^{T} \tilde{W}(t)-\frac{1}{2}\|\theta\|^{2}(T-t)\right) \mathbb{P} .
$$

The process $\tilde{W}(t)$ is a Brownian motion in $[0, T]$.

By Girsanov's theorem, we have

$$
X(t)=x+\int_{0}^{T} r_{R}(s) X(s) d s+\int_{0}^{T} c(s) d s+\int_{0}^{T} \mathcal{U}(s) \Sigma X(s) d \tilde{W}(s)
$$

where $\Sigma=\left(\begin{array}{cc}\sigma_{S}^{S} & \sigma_{S}^{I} \\ 0 & \sigma_{I} \theta_{I}\end{array}\right)$

Taking the expectation with respect to the filtration $\mathcal{F}_{t}$, we obtain

$$
\begin{aligned}
\mathbb{E}_{t}[X(t)]=x & +\mathbb{E}_{t}\left[\int_{0}^{T} r_{R}(s) X(s) \mid \mathcal{F}_{t}^{I}, \mathcal{F}_{t}^{S}\right] d s+\mathbb{E}_{t}\left[\int_{0}^{T} c(s) \mid \mathcal{F}_{t}^{I}, \mathcal{F}_{t}^{S}\right] d s \\
& +\mathbb{E}_{t}\left[\int_{0}^{T} \mathcal{U}(s) \Sigma X(s) d \tilde{W}(s) \mid \mathcal{F}_{t}^{I}, \mathcal{F}_{t}^{S}\right]
\end{aligned}
$$


But the real interest rate and the contribution processes are independent of the filtration $\left\{\mathcal{F}_{t}^{S}, \mathcal{F}_{t}^{S}\right\}$, hence

$$
\mathbb{E}_{t}[X(t)]=x+\int_{0}^{T} r_{R}(s) \mathbb{E}_{t}\left[X(s) \mid \mathcal{F}_{t}^{I}, \mathcal{F}_{t}^{S}\right] d s+\int_{0}^{T} c(s) d s
$$

For any deterministic function $h(s)=\mathbb{E}_{t}\left[X(s) \mid \mathcal{F}_{t}^{I}, \mathcal{F}_{t}^{S}\right]$ in $[0, T]$ similarly satisfies the ordinary differential equation $X(t, T, \mathcal{U}(\cdot), x)$ on $[t, T]$. It can also be observed that

$$
X(t, T, \mathcal{U}(\cdot), x) \geq H(t), \quad \text { on }[0, T]
$$

In conclusion, it is easy to see that, when $\mathcal{U}(\cdot) \equiv 0$ the deterministic equation (34) yields;

$$
r_{R}(t) X(t)+c(t) \geq 0
$$

As $H(t)$ is the minimal guarantee then,

$$
r_{R}(t) H(t)+c(t) \geq 0
$$

which completes the proof.

Note that from lemma (2), the negative sign associated with the arbitrary contribution done by the clients simply shows that the money is withdrawn from the client.

\section{Optimization Problem}

The manager has the expected terminal minimum target $H_{T}$ to reach at the end of the his client's retirement. The manager has to maximize the surplus amount denoted as $X(T)-H_{T}$ where his benefit lies. A certain proportion of the surplus that the manager would acquire at the end is $\beta\left(X(T)-H_{T}\right.$ ), as in (Deelstra et al, 2000). The program manager desires to maximize the expected utility of his terminal wealth over the admissible controls, $\mathcal{U}_{a d}(\cdot)$, that is;

subject to

$$
\sup _{\left\{u_{S}(t), u_{B}(t)\right\} \in \mathcal{U}_{a d}(\cdot)} E_{t}\left[U\left(X(T)-H_{T}\right)\right]
$$

$$
\begin{array}{r}
d X(t)=\left(\left[r_{R}(t)+\left(\begin{array}{lc}
u_{S}(t) & \left.u_{B}(t)\right)
\end{array}\right)\left(\begin{array}{c}
\lambda_{1} \sigma_{S}^{S}+\lambda_{2} \sigma_{S}^{I} \theta_{I} \\
\sigma_{I} \theta_{I}
\end{array}\right)\right] X(t)+c(t)\right) d t \\
+\left(\begin{array}{ll}
u_{S}(t) & \left.u_{B}(t)\right)
\end{array}\right)\left(\begin{array}{cc}
\sigma_{S}^{S} & \sigma_{S}^{I} \\
0 & \sigma_{I} \theta_{I}
\end{array}\right)\left(\begin{array}{l}
d W_{S}(t) \\
d W_{I}(t)
\end{array}\right) X(t)+u_{B}(t) \sigma^{I} X(t) d W_{I} \\
u_{S}(t) \geq 0 \\
u_{B}(t) \geq 0 \\
1-u_{S}(t)-u_{B}(t) \geq 0
\end{array}
$$

The above problem is a constrained optimization problem. Introducing the value function for the optimization problem, we have;

$$
V(t, X(t))=\sup _{\left\{u_{S}(t), u_{B}(t)\right\} \in \mathcal{U}_{a d}(\cdot)} E_{t}\left[U\left(\left(X(T)-H_{T}\right) \mid X(t)=X\right]\right.
$$

Assumption 1. Let the utility function be a smooth non-negative, increasing concave function, that is

$$
U(V(t, X)) \in C^{1,2}([0, T] \times \mathbb{R}) .
$$

The value function is continuous, once differentiable in $t$ and twice differentiable with respect to $X(t)$ according to assumption (1). Then by Itô-Doeblin lemma and using relation in equation (9) to the value function, we obtain the Hamilton-Jacobi-Bellmann (HJB) equation of the form;

$$
\begin{aligned}
& 0=\frac{\partial V}{\partial t}+\sup _{\left\{u_{S}(t), u_{B}(t)\right\} \in \mathcal{U}_{a d}(\cdot)}\left\{\left[\left[r_{R}(t)+\left(\begin{array}{ll}
u_{S}(t) & \left.u_{B}(t)\right)
\end{array}\right)\left(\begin{array}{c}
\lambda_{1} \sigma_{S}^{S}+\lambda_{2} \sigma_{S}^{I} \theta_{I} \\
\sigma_{I} \theta_{I}
\end{array}\right)\right] X(t)\right.\right. \\
& +c(t)] \frac{\partial V}{\partial X}+\frac{1}{2}\left[\left(\begin{array}{ll}
u_{S}^{2}(t) & u_{B}^{2}(t)
\end{array}\right)\left(\begin{array}{c}
\left(\sigma_{S}^{S}\right)^{2}+\left(\sigma_{S}^{I}\right)^{2}+\sigma_{S}^{I} \sigma_{S}^{S} \rho_{S I} \\
\sigma_{I}^{2} \theta_{I}^{2}
\end{array}\right)\right. \\
& \left.\left.+u_{S}(t) u_{B}(t)\left[2\left(\sigma_{S}^{I}\right)^{2}+\sigma_{S}^{S} \sigma_{S}^{I} \rho_{S I}\right]\right] X^{2}(t) \frac{\partial^{2} V}{\partial X^{2}}\right\}
\end{aligned}
$$


with the initial and terminal conditions,

$$
V\left(0, x_{0}\right)=V_{0}, \quad V(T, X(T))=X(T)-H_{T}
$$

To find the optimal proportions to be invested in the risky assets, we take the first derivative with respect to each optimal policy. Therefore, the optimal proportion to be invested in the inflation-linked bond is,

$$
u_{B}(t)=-\frac{\left(\left(\sigma_{S}^{I}\right)^{2}+\frac{1}{2} \rho_{S I} \sigma_{S}^{S} \sigma_{S}^{I}\right)}{\left(\sigma_{I} \theta_{I}\right)^{2}} u_{S}(t)-\frac{1}{\sigma_{I} \theta_{I}} \frac{\frac{\partial V}{\partial X}}{X(t) \frac{\partial^{2} V}{\partial X^{2}}}, \quad \sigma_{I} \theta_{I} \neq 0 .
$$

while proportion to be invested in the stock price given by,

$$
\begin{aligned}
u_{S}(t) & =-\frac{\left(\left(\sigma_{S}^{I}\right)^{2}+\frac{1}{2} \rho_{S I} \sigma_{S}^{S} \sigma_{S}^{I}\right)}{\left[\left(\sigma_{S}^{S}\right)^{2}+\left(\sigma_{S}^{I}\right)^{2}+\sigma_{S}^{S} \sigma_{S}^{I} \rho_{S I}\right]} u_{B}(t) \\
& -\frac{\left(\lambda_{1} \sigma_{S}^{S}+\lambda_{2} \sigma_{S}^{I} \theta_{I}\right)}{\left[\left(\sigma_{S}^{S}\right)^{2}+\left(\sigma_{S}^{I}\right)^{2}+\sigma_{S}^{S} \sigma_{S}^{I} \rho_{S I}\right]} \frac{\frac{\partial V}{\partial X}}{X(t) \frac{\partial^{2} V}{\partial X^{2}}} .
\end{aligned}
$$

such that $\left(\sigma_{S}^{S}\right)^{2}+\left(\sigma_{S}^{I}\right)^{2}+\sigma_{S}^{S} \sigma_{S}^{I} \rho_{S I} \neq 0$

It is observed that the two investment strategies are given in-terms of each other. The two equations, (42) and (43) are solved simultaneously to get the optimal investment proportions. Before simultaneously solving the the two equations, for simplicity let

$$
\begin{array}{r}
A=\left(\sigma_{S}^{I}\right)^{2}+\frac{1}{2} \rho_{S I} \sigma_{S}^{S} \sigma_{S}^{I} \\
B=\left(\sigma_{S}^{S}\right)^{2}+\left(\sigma_{S}^{I}\right)^{2}+\sigma_{S}^{S} \sigma_{S}^{I} \rho_{S I} \\
C=\lambda_{1} \sigma_{S}^{S}+\lambda_{2} \sigma_{S}^{I} \theta_{I} \\
D=\sigma_{I} \theta_{I}
\end{array}
$$

Therefore,

$$
\begin{aligned}
u_{B}^{*}(t) & =\frac{\left[\frac{1}{D}+\frac{A C}{B D^{2}}\right]}{\left[1-\frac{A^{2}}{B D^{2}}\right]} \frac{\frac{\partial V}{\partial X}}{X(t) \frac{\partial^{2} V}{\partial X^{2}}} \\
& =\left[\frac{B D-A C}{B D^{2}-A^{2}}\right] \frac{\frac{\partial V}{\partial X}}{X(t) \frac{\partial^{2} V}{\partial X^{2}}}
\end{aligned}
$$

and on the stock as

$$
u_{S}^{*}(t)=\frac{D[C D+A]}{\left[B D^{2}-A^{2}\right]} \frac{\frac{\partial V}{\partial X}}{X(t) \frac{\partial^{2} V}{\partial X^{2}}}
$$

Substituting equations (44) and (45) back to the partial differential equation (PDE) (41) in-order to find the value function, it yields the following equation

$$
\begin{aligned}
\frac{\partial V}{\partial t} & +\left[r_{R}(t) X(t)+c(t)\right] \frac{\partial V}{\partial X} \\
& +\frac{1}{B D^{2}-A^{2}}[C(C D+A)+(A B C D)] \frac{\left(\frac{\partial V}{\partial X}\right)^{2}}{\left(\frac{\partial^{2} V}{\partial X^{2}}\right)} \\
& +\frac{1}{2\left(B D^{2}-A^{2}\right)^{2}}[B D(C D+A)+A(C D+A)(B D-A C) \\
& \left.+D^{2}(B D-A C)\right] \frac{\left(\frac{\partial V}{\partial X}\right)^{2}}{\left(\frac{\partial^{2} V}{\partial X^{2}}\right)}=0
\end{aligned}
$$


The equation (46) is simplifies to a nonlinear second order partial differential equation of the form:

$$
\frac{\partial V}{\partial t}+\left[r_{R}(t) X(t)+c(t)\right] \frac{\partial V}{\partial X}+G^{*} \frac{\left(\frac{\partial V}{\partial X}\right)^{2}}{\left(\frac{\partial^{2} V}{\partial X^{2}}\right)}=0 .
$$

where

$$
\begin{aligned}
G^{*}= & \frac{1}{B D^{2}-A^{2}}[C(C D+A)+(A B C D)]+\frac{1}{2\left(B D^{2}-A^{2}\right)^{2}} \\
& {\left[B D(C D+A)+A(C D+A)(B D-A C)+D^{2}(B D-A C)\right] }
\end{aligned}
$$

The above equation is difficult to solve in that format. For the explicit solutions, we would consider the pension fund manager's objective function.

\section{Explicit Solution in CRRA Utility Case}

Suppose the utility value function that pension fund manager aspires to maximize his/her surplus over is given by

$$
\begin{aligned}
U(V(t, X(t))) & =V(t, X(t)) \\
& =\frac{(X(t)-H(t))^{\gamma}}{\gamma}, \quad \gamma \in(-\infty, 0) \cup(0,1)
\end{aligned}
$$

where $H(t)$ is defined as in subsection (2.3).

Theorem 1 Suppose the utility function is given as in equation (49), then

(i) the wealth process is proportional to the minimum guarantee process given as

$$
X(t)=\frac{X_{0}}{H_{0}} H(t) \exp \left\{-\left(\frac{a+1}{a}\right) \int_{0}^{t} r_{R}(s) d s\right\}
$$

where

$$
H(t)=H_{0} \exp \left[\frac{G^{*}}{1-\gamma} t+\frac{1}{a} \int_{0}^{t} r_{R}(s) d s\right], \quad t \in[0, T]
$$

for a 0 be a proportionality constant.

(ii) the optimal investment portfolio values in the stock market price and the inflation-linked bond are respectively given as,

$$
\begin{gathered}
u_{S}^{*}(t)=\frac{D[C D+A]}{(1-\gamma)\left[B D^{2}-A^{2}\right]}\left[\frac{H_{0}}{X_{0}} \exp \left[\left(\frac{a+1}{a}\right) \int_{0}^{t} r_{R}(s) d s\right]-1\right] \\
u_{B}^{*}(t)=\left[\frac{B D-A C}{(1-\gamma)\left[B D^{2}-A^{2}\right]}\right]\left[\frac{H_{0}}{X_{0}} \exp \left[\left(\frac{a+1}{a}\right) \int_{0}^{t} r_{R}(s) d s\right]-1\right]
\end{gathered}
$$

proof.

(i) From the utility function given in equation (49),

$$
V(t, X(t))=\frac{(X(t)-H(t))^{\gamma}}{\gamma}, \quad \gamma \in(-\infty, 0) \cup(0,1)
$$

Taking the partial derivatives of equation 47 , we have:

$$
\begin{aligned}
\frac{\partial V}{\partial t} & =(X(t)-H(t))^{\gamma-1}[d X(t)-d H(t)] \\
\frac{\partial V}{\partial X} & =(X(t)-H(t))^{\gamma-1} \\
\frac{\partial^{2} V}{\partial X^{2}} & =(\gamma-1)(X(t)-H(t))^{\gamma-2} .
\end{aligned}
$$


Substituting the above partial derivatives of the value function in equation (47), we get

$$
\begin{array}{r}
(X(t)-H(t))^{\gamma-1}[d X(t)-d H(t)]+\left[r_{R}(t) X(t)+c(t)\right] \\
(X(t)-H(t))^{\gamma-1}+\frac{G^{*}}{(\gamma-1)}(X(t)-H(t))^{\gamma}=0 .
\end{array}
$$

Expanding equation (57),

$$
\begin{array}{r}
(X(t)-H(t))^{\gamma-1} d X(t)-(X(t)-H(t))^{\gamma-1} d H(t)+\left[r_{R}(t) X(t)+c(t)\right] \\
(X(t)-H(t))^{\gamma-1}+\frac{G^{*}}{(\gamma-1)}(X(t)-H(t))^{\gamma}=0
\end{array}
$$

Dividing equation (58) by $(X(t)-H(t))^{\gamma-1}$ and by separating the equation into two equations, we have:

$$
d X(t)+r_{R}(t) X(t)-\frac{G^{*}}{1-\gamma} X(t)=0
$$

and

$$
d H(t)-\frac{G^{*}}{1-\gamma} H(t)-c(t)=0
$$

Equation (59) is a first order ordinary differential equation whose solution is

$$
X(t)=X_{0} \exp \left[\frac{G^{*}}{1-\gamma} t-\int_{0}^{t} r_{R}(s) d s\right], \quad t \in[0, T]
$$

To solve equation (60), we use the relationship between the expected minimum guarantee process established in lemma (), that

$$
r_{R}(t) H(t) \geq-c(t), \quad \forall t \in[0, T]
$$

Let $a<0$ be a proportionality constant to the inequality raised above, such that

$$
r_{R}(t) H(t)=a c(t), \quad \forall t \in[0, T]
$$

Substituting equation (62) into equation (60), yields another first order ODE of the form:

$$
d H(t)=\frac{G^{*}}{1-\gamma} H(t)+\frac{1}{a} r_{R}(t) H(t)
$$

whose solution is given as,

$$
H(t)=H_{0} \exp \left[\frac{G^{*}}{1-\gamma} t+\frac{1}{a} \int_{0}^{t} r_{R}(s) d s\right], \text { for } t \in[0, T]
$$

Looking at the two equations (61) and (64), it is seen that indeed the wealth is proportional to the expected minimum guarantee process given as,

$$
X(t)=\frac{X_{0}}{H_{0}} H(t) \exp \left[-\left(\frac{a+1}{a}\right) \int_{0}^{t} r_{R}(s) d s\right], \text { for } t \in[0, T] .
$$

which completes the first part of the theorem.

(ii) From equations (45) and (44), we respectively have,

$$
u_{S}^{*}(t)=\frac{D[C D+A]}{\left[B D^{2}-A^{2}\right]} \frac{\frac{\partial V}{\partial X}}{X(t) \frac{\partial^{2} V}{\partial X^{2}}}
$$




$$
u_{B}^{*}(t)=\left[\frac{B D-A C}{B D^{2}-A^{2}}\right] \frac{\frac{\partial V}{\partial X}}{X(t) \frac{\partial^{2} V}{\partial X^{2}}}
$$

Substituting in the partial derivatives of the value function and simplifying we get,

$$
u_{S}^{*}(t)=\frac{D[C D+A]}{\left[B D^{2}-A^{2}\right]} \frac{(X(t)-H(t))}{(\gamma-1) X(t)}
$$

as the investment portfolio in the stock market, while for the inflation-linked bond is

$$
u_{B}^{*}(t)=\left[\frac{B D-A C}{B D^{2}-A^{2}}\right] \frac{(X(t)-H(t))}{(\gamma-1) X(t)}
$$

Using the results of theorem (1)(i), we finally obtain the results that, the optimal investment portfolio value in the stock market price is

$$
u_{S}^{*}(t)=\frac{D[C D+A]}{(1-\gamma)\left[B D^{2}-A^{2}\right]}\left[\frac{H_{0}}{X_{0}} \exp \left[\left(\frac{a+1}{a}\right) \int_{0}^{t} r_{R}(s) d s\right]-1\right]
$$

and in the inflation-linked bond is given by

$$
u_{B}^{*}(t)=\left[\frac{B D-A C}{(1-\gamma)\left[B D^{2}-A^{2}\right]}\right]\left[\frac{H_{0}}{X_{0}} \exp \left[\left(\frac{a+1}{a}\right) \int_{0}^{t} r_{R}(s) d s\right]-1\right]
$$

It has been assumed that the proportionality constant $(a)$ should attain any value less than zero provided the equality in lemma (2) is attained. Therefore, looking at theorem (1) we have the corollary.

\section{Corollary 1}

(i) For $-1<a<0$, the wealth process represents the Growth function.

(ii) For $a<-1$, the wealth represents a decay function.

\section{Numerical Example}

Suppose the inflation rate is currently estimated at $3 \%$ and the client has twenty years before reaching the his or her retirement period. The real interest rate is annually compounded at $2 \%$, with mean one and mean reversion parameter 0.5 . The supplementary contribution that the client has to make to amortize the fund is $2 \%$ of the fixed contribution from the salary. By using the parameters of the numerical example of (Nkeki \& Nwozo, 2013), the figures below were obtained. That is; the growth of the stochastic real interest rate, the graph showing how the client would make supplementary contributions for the twenty years period. The last two figures depicts how the investor should optimally invest in the portfolios.

\section{Discussion}

Note in figures (3) and (4) below, $u_{A}^{*}$ denotes the optimal portfolio allocation onto the cash account and furtherly our discussion is based on the numerical example given in previous section to extrapolate our results.

Figure (1) shows how the real interest rate would evolve with in twenty years period time. Therefore, the investor with higher risk aversion coefficient should invest less in the stock and the inflation linked bond at the initial stage. The real interest rate increases significantly after ten years, that would be the right time for the investor to invest more on both risky assets.

Figure (2) shows that the supplementary contribution with time approaches zero. This is due to the fact that past and present imbalances by the retirement age they will be met. It can be noted that at after ten years a small amount will be required to amortize the fund. The is will be subsidized by the high interest rates. Taking a keen look at our work, it shows that supplementary contribution follows what (Gao, 2009) deem the correction factor. 


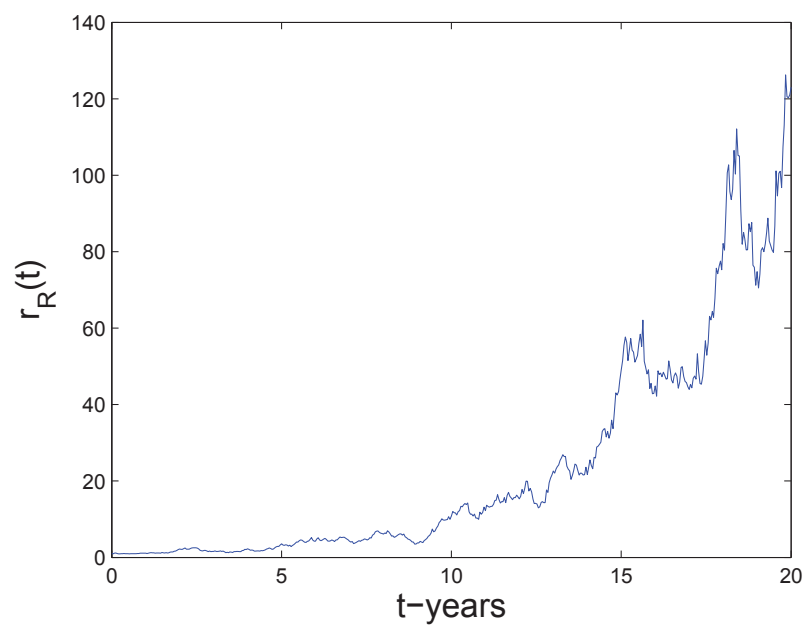

Figure 1: The real interest rate $\left(r_{R}(t)\right.$ over a period of 20 years.

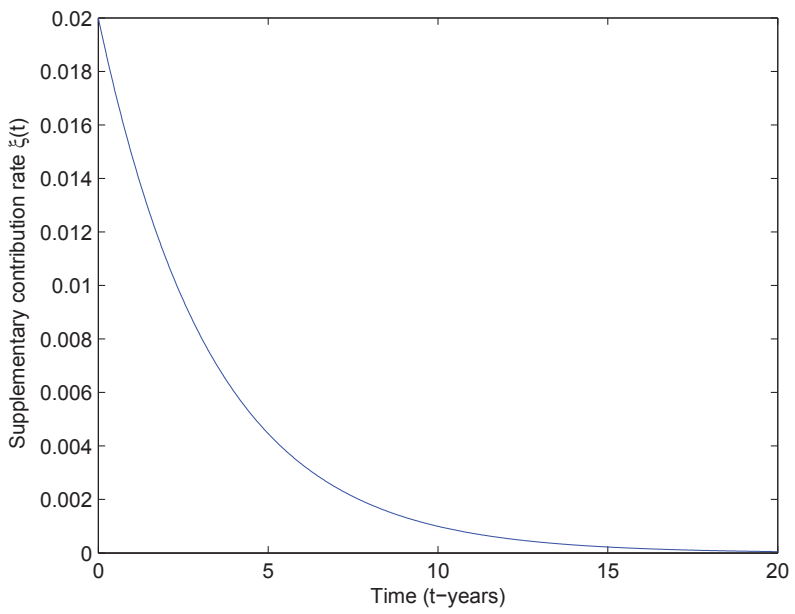

Figure 2: The contribution rate paid by the client to amortize past experiences 20 years.

Considering figure (3), the value of the parameter $a$ plays an important role on the portfolio that the investor has to choose in order to optimize his rewards. The first graph shows that the investor from the onset may choose any portfolio or both portfolios for a period of 10 years. Then after 10 years he should invest more on stock market and less on the cash account which has a smaller gain. The investor should completely not invest the inflation linked bond as that will attract negative interest rates. The same trend is observed in all the other graphs except the last with increase in the value of investment. In the last graph for $a<-1$, it is wise to invest all the resources in the risk-less portfolio for a period of at least 15 years, then he may invest in the stock market.

Figure (4) shows that whenever the risk averse parameter $(\gamma \leq 0)$, the intuitive conclusion is that all the portions of the stock and bonds should be replaced by cash for the investor to hit his maximal rewards as evidenced by the first graph. As for less risk averse investors $(0<\gamma<1)$, the more initially the investor invest in the cash account and gradually increase the and stock investments and decreasing the bond the better. However, for small parameters of the risk averse parameter $(0<\gamma \leq 0.1)$, it is more risky to invest in both the stock and the bond. It is advisable for the investor to consider the cash account but may at times take risks with stock market though it is risky. 

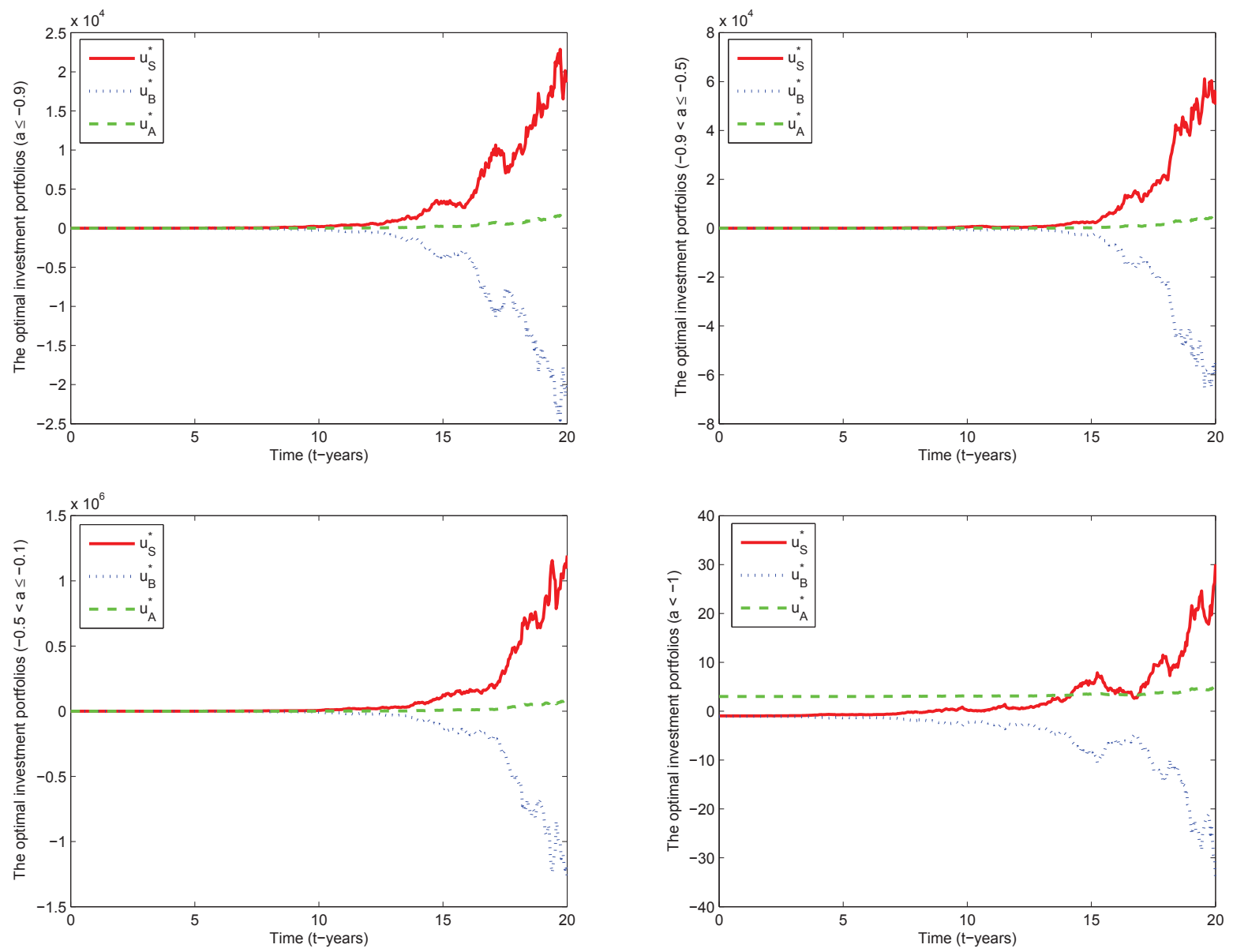

Figure 3: The graphs shows how the investor should pursue portfolio allocations, at different values of the proportionality parameter $a$. The value of $\gamma$ was kept at 0.5 throughout.

\section{Conclusion and Future Work}

The optimal investment strategies that an investor should partake in a DC pension fund have been investigated. The risky assets, the stock price and the inflation-linked bond are described by the GBM. The method of stochastic optimal control and by application of the maximum principle to the optimization problem, the wealth process, minimum guarantee and admissible controls were attained. The solutions were obtained through the help of the CRRA utility function that the investor aspired to maximize upon. The minimum guarantee process played a very important role in guiding the investor as evidenced from theorem (1). The contribution process which was in two forms being the fixed amount agreed between the employer and pension scheme and the supplementary contribution done by the client towards his or her fund lead to some interesting results as given by both lemma (1) and (2). The value of the proportionality parameter $(a)$, is of paramount importance as given in corollary (1), with respect to the relationship in lemma (2). In future the same model maybe be applied to the constant absolute risk aversion (CARA) utility function. The model may be extended by introducing time dependent volatility parameters.

\section{Acknowledgements}

My sincere gratitude goes to Professor Xue for his guidance towards the write-up of this manuscript. Special thanks are extended to the Chinese Government Scholarship Council for giving us an opportunity to pursue this research, without forgetting the unknown reviewers of this manuscript for their valuable comments. 

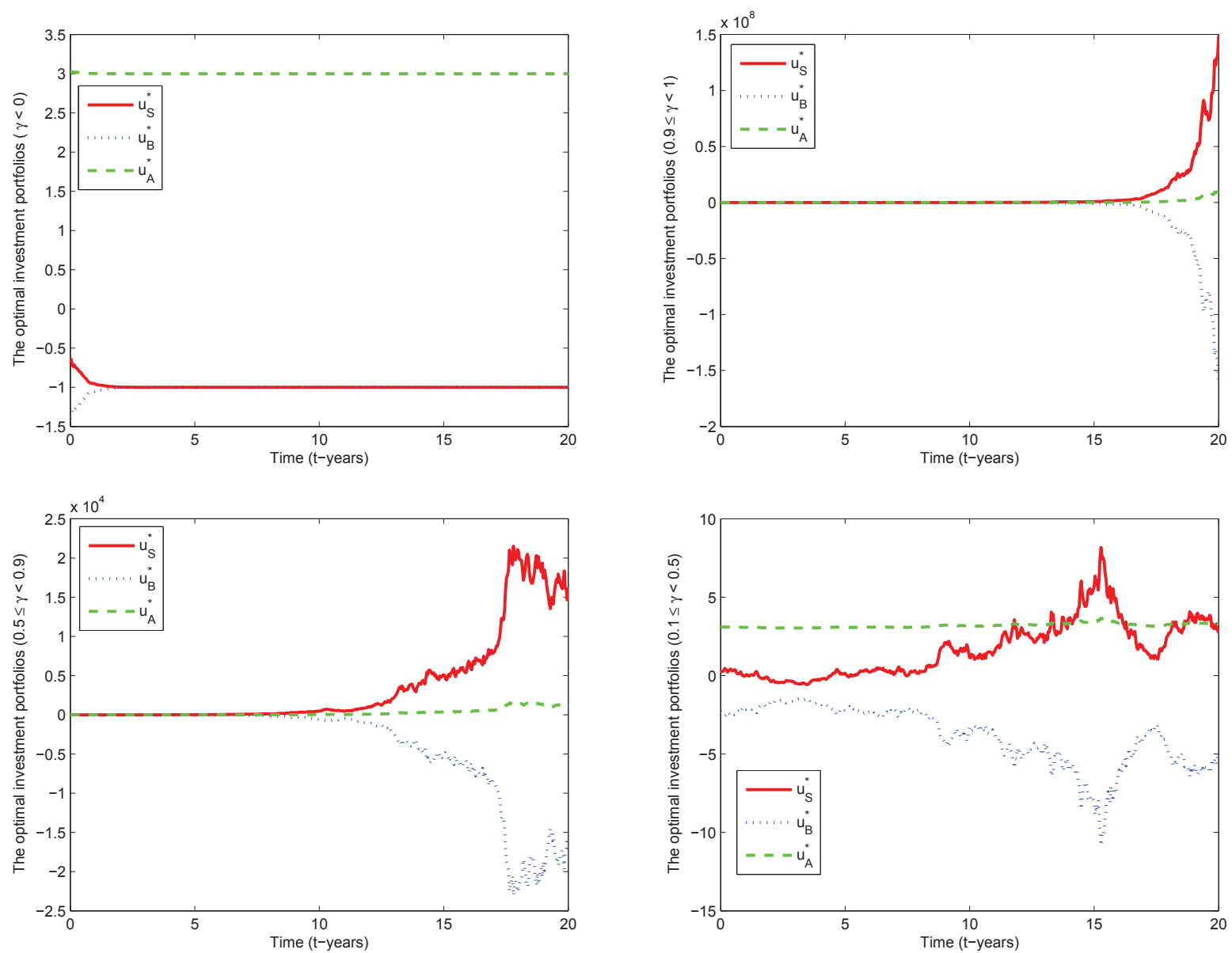

Figure 4: The graphs shows how the investor should pursue portfolio allocations, at different values of the risk aversion parameter $\gamma$. The value of $a$ was kept at -0.5 throughout.

\section{References}

Beletski, T. (2006). Inflation-linked products and optimal investment with macro derivatives: Unpublished thesis. http://kluedo. ub. uni-kl. de/volltexte/2006/2045/index. html

Blake, D. (1998). Pension schemes as options on pension fund assets Insurance: Mathematics and Economics, Vol. 23, (pp 263-286)., Elsevier. http://dx.doi.org/10.1016/S0167-6687(98)00048-1

Bodie, Z., Kane, A., \& Marcus, A. J. (2011). Investments and portfolio management McGraw-Hill/Irwin. https://compass2g.illinois.edu

Cairns, A. J. G., Blake, \& Dowd, K. (2006). Stochastic lifestyling: Optimal dynamic asset allocation for defined contribution pension plans. Journal of Economic Dynamics and Control, Vol 30. (pp 843-877), Elsevier. http://dx.doi.org/10.1016/j.jedc.2005.03.009

Boulier, J., Huang, S. \& Taillard, G. (2001). Optimal management under stochastic interest rates: the case of a protected defined contribution pension fund Insurance: Mathematics and Economics, Vol. 28, (pp. 173-189), Elsevier.http://dx.doi.org/10.1016/S0167-6687(00)00073-1

Deelstra, G., Grasselli, M \& Koehl, P. (2003). Optimal investment strategies in the presence of a minimum guarantee. Insurance: Mathematics and Economics, Vol. 1633, (pp. 189-207), Elsevier.

http://dx.doi.org/10.1016/S0167-6687(03)00153-7 
Deelstra, G., Grasselli, M., Koehl, P. çois and others. (2000). Optimal investment strategies in a CIR framework. Journal of Applied Probability., Vol. 37, (pp. 936-946). Applied Probability Trust. http://projecteuclid.org/euclid.jap/1014843074

Devolder, P., Bosch P. M. \& Dominguez, F. I. (2003). Stochastic optimal control of annuity contracts. Insurance: Mathematics and Economics., Vol. 33, (pp. 227-238). Elsevier. http://dx.doi.org/10.1016/S01676687(03)00136-7

Fisher, I \& others. (1930). The theory of interest. Macmillan New York. http://www.sfu.ca/ easton/MA-IL806/Fisher

Gao, J. (2009). Optimal portfolios for DC pension plans under a CEV model. Insurance: Mathematics and Economics., Vol. 44, (pp. 479-490). Elsevier. http://dx.doi.org/10.1016/j.insmatheco.2009.01.005

Haberman, S \& Vigna, E. (2002). Optimal investment strategies and risk measures in defined contribution pension schemes. Insurance: Mathematics and Economics., Vol. 31, (pp. 35-69). Elsevier. http://dx.doi.org/10.1016/S0167-6687(02)00128-2

Nkeki, C. I. \& Nwozo, C. R. (2013). Optimal Investment under Inflation Protection and Optimal Portfolios with Stochastic Cash Flows Strategy. International Journal of Applied Mathematics., Vol. 43. IJAM_43_2_02

Nkeki, C. I. \& Nwozo, C. R. (2012). Variational form of classical portfolio strategy and expected wealth for a defined contributory. Journal of Mathematical Finance: Scientific Research Publishing. http://dx.doi.org/10.4236/jmf.2012.21015

Vigna, E \& Haberman, S. (2001). Optimal investment strategy for defined contribution pension schemes. Insurance: Mathematics and Economics., Vol. 28, (pp. 233-262). Elsevier. http://dx.doi.org/10.1016/S01676687(00)00077-9

Wilkie, A Dv. (1985). Portfolio selection in the presence of fixed liabilities: A comment on the matching of assets to liabilities. Journal of the Institute of Actuaries., Vol. 28, (pp. 229-277). Cambridge Univ Press. http://dx.doi.org/10.1017/S0020268100042128

Zhang, A., Korn, R. \& Ewald, C. O. (2007). Optimal management and inflation protection for defined contribution pension plans. Blätter der DGVFM., Vol. 28, (pp. 239-258). Springer. http://dx.doi.org/10.1007/s11857007-0019-x

\section{Copyrights}

Copyright for this article is retained by the author(s), with first publication rights granted to the journal.

This is an open-access article distributed under the terms and conditions of the Creative Commons Attribution license (http://creativecommons.org/licenses/by/3.0/). 